\title{
Use of a Shock Tube Platform in the Replication of Blast Lung Injury
}

\author{
Benjamin Butler', Thuy-Tien Nguyen ${ }^{2}$, Alun Williams ${ }^{3}$, Andrew Tucker $^{3}$, William G. \\ Proud $^{2,4}$ and Katherine A. Brown ${ }^{1,5 *}$ \\ ${ }^{1}$ Cavendish Laboratory, Department of Physics, University of Cambridge, JJ Thomson Avenue, \\ Cambridge CB3 0HE, UK \\ ${ }^{2}$ Department of Bioengineering, Imperial College London, London SW7 2AZ, UK \\ ${ }^{3}$ Department of Veterinary Medicine, University of Cambridge, Madingley Road, Cambridge CB3 \\ OES, UK \\ ${ }^{4}$ Institute of Shock Physics, Department of Physics, Imperial College London, London SW7 2AZ, UK \\ ${ }^{5}$ Department of Molecular Biosciences, The University of Texas at Austin, Austin, TX 78712, USA
}

\begin{abstract}
War and asymmetrical conflicts are becoming increasingly prevalent in the modern world. Due to improvements in conflict medicine, survivable injuries are now more severe than they once were. Therefore, it is now more important than ever that there exist scientific and engineering methods for replicating wartime injuries in the context of the laboratory. We have developed one such method: a shock tube platform for testing ex vivo samples of the porcine respiratory system. Using this platform, we can, to some extent, simulate the pathophysiological consequences of blast lung. This is a condition commonly present in victims of explosive blasts, both those due to typical armaments and Improvised Explosive Devices (IEDs). Presented here are the results of experiments conducted using porcine bronchiole tissue as ex vivo organ cultures. Data presented show epithelial damage, consistent with known trauma-induced cell injury that can lead to acute respiratory distress syndrome (ARDS).
\end{abstract}

\section{Introduction}

Primary blast lung injury is a fundamental component of life-threatening blast injuries [1,2]. Damage to lung tissue is believed to occur as a result of the generation of shear and stress waves from energy dissipation of a blast wave [3]. Blast lung is an example of a primary type of blast injury, which arises directly from the blast wave and not from other causes (e.g., projectiles, physical impacts, chemicals, fire) that may also be associated with the blast [4].

Blast lung is typically characterized by haemorrhage and injury of functional respiratory tissue, known as parenchyma, including the rupture of lower airway air sacs called alveoli [2]. Blast damage to the respiratory system is not always accompanied by obvious symptoms and can, in some cases, be delayed by as much as 32 hours during which an inflammatory response develops $[1,2,4]$. Depending on the severity of the injury and accompanying inflammation, the life-threatening acute respiratory distress syndrome (ARDS) can arise

* Corresponding author: kb518@,cam.ac.uk 
$[1,2]$. No specific therapy exists for primary shock wave induced injury to respiratory tissues and treatment is primarily supportive and can require mechanical ventilation depending upon the severity of the clinical diagnosis (e.g., ARDS), and the presence of other life-threatening injuries $[1,2]$.

There is considerable interest in understanding short and long-term pathological changes in tissue arising from pressure wave-associated traumas that affect the respiratory systems [1,5]. A key goal of this research is to establish a better understanding of the mechanical, biological and pathological effects induced by pressure waves over a variety of length-scales (e.g., from cells to whole organisms), which in turn demands high-quality experimental models. In this context, we are exploring the use of shock tube systems for creating new models of soft tissue injury and trauma. Here we report shock tube studies aimed at recapitulating respiratory injuries associated with blast lung using porcine bronchiole tissue ex vivo organ cultures (EVOCs).

\section{Methods}

Samples were obtained and prepared following previously described procedures [6]. Briefly, isogenic bronchiole tissues were obtained from six- to eight-week-old piglets sacrificed by intravenous administration of sodium pentobarbitone. The respiratory system of the animals was removed, and bronchiole tissues were isolated. The tissues were then submerged in liquid media (Dulbecco's Modified Eagle Medium, supplemented with a penicillin/streptomycin mix) and stored in a $37^{\circ} \mathrm{C}, 5 \% \mathrm{CO}_{2}$ incubator. After approximately two hours and then again after four hours of washing, the media was replaced. Full thickness tissue samples were taken as circular discs using a 4-mm diameter biopsy punch and placed on agarose plug held in a plastic well containing Phosphate Buffered Saline (PBS) prior to testing.

Samples were subjected to a high-pressure pulse from the shock tube in a modified, low impulse configuration [7]. Control EVOCs were placed in the sample holder of the shock tube (Fig. 1), and then removed and prepared for histological analysis. Additional EVOCs were placed in the sample holder and subjected to pressure waves generated in the shock tube at diaphragm burst pressures of 2.19 bar and then immediately removed and prepared for histological analysis. The propagation and evolution of produced blast waves are monitored by piezoelectric dynamic pressure sensors (Dytran ${ }^{\circledR} 2300$ V1 Low Impedance Voltage Mode - LIVM) as previously described [8].

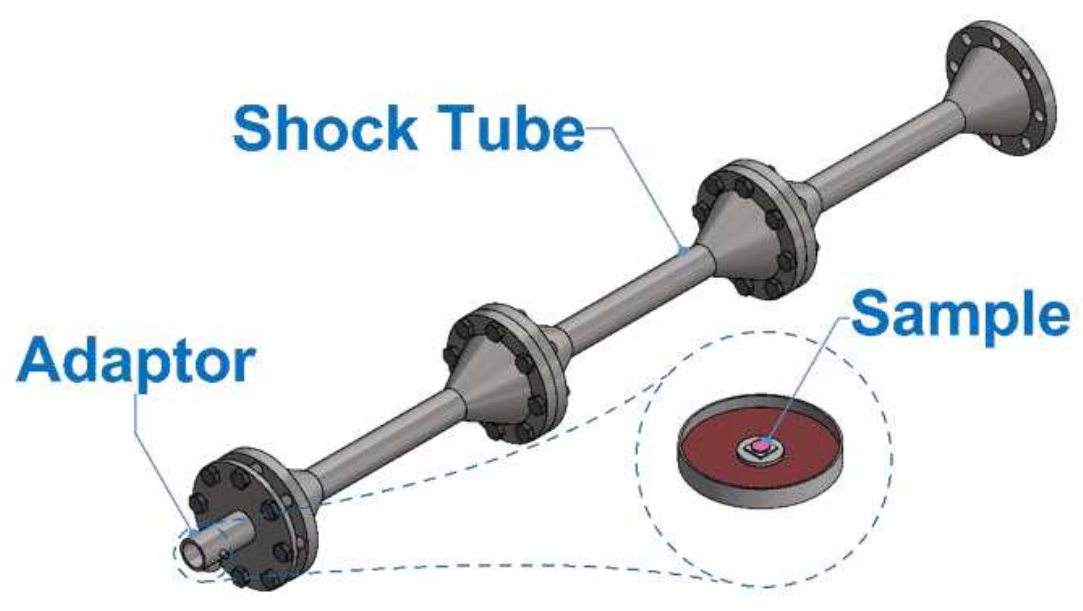

Fig. 1. Schematic image of the shock tube used in this study. It is comprised of a driving tube, a driven tube and a custom-made adaptor in which samples are held in place using a threaded screw. A shock wave resulting from the rupture of a membrane held at the interface between the driving and driven tubes propagates through the system and over the sample. A pressure sensor present in the central flanges of the driven tube monitors the wave. 


\section{Results}

Fig. 2 shows optical histology images of a control and a pressure wave-subjected porcine bronchiole EVOC with its accompanying pressure profile. The control sample (Fig. 2A) demonstrating normal histological features including the presence of structurally intact epithelium on the outer apical surface. The EVOC shown in Fig. 2B exhibited histological changes demonstrating damage to the apical epithelial layer, indicated by desquamation and the presence of cell debris. This type of tissue damage, arising from the pressure pulse shown in Fig. 2C, is consistent with previously reported observations of tracheal tissue subjected to blast-type pressures [9].
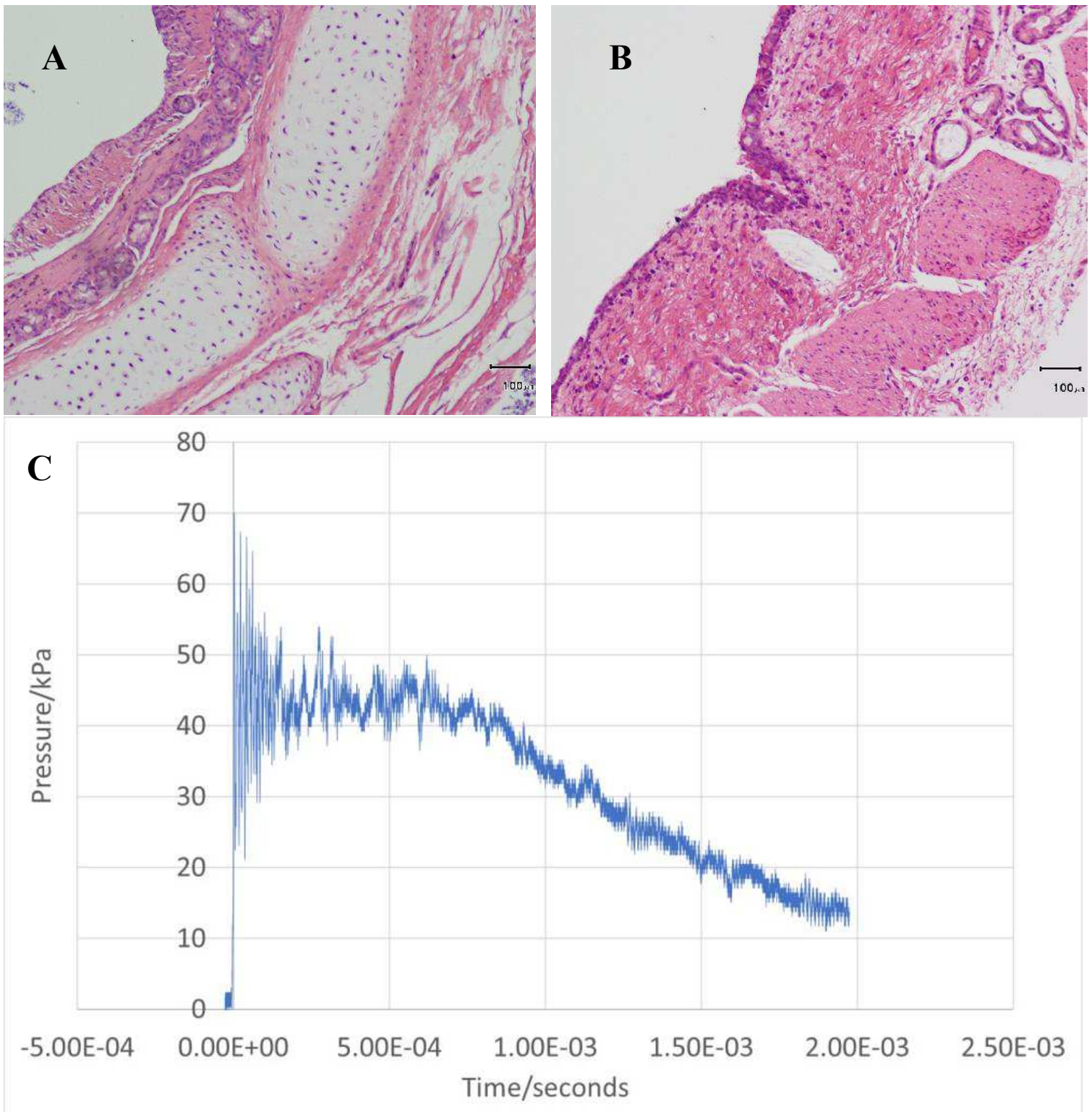

Fig. 2 Comparative histological images of bronchiole EVOC samples together with a corresponding pressure trace. (A) Control sample that has undergone identical processing to test samples without having experience a pressure pulse (sham). (B) Sample was exposed to a pressure pulse with a diaphragm burst pressure of 2.19 bar. At this pressure, some parts of the topmost epithelial layer are sloughed off (evidence of desquamation) compared to the sham sample. (C) Pressure trace, as measured by a piezoelectric sensor, of the experiment on the sample shown in (B). 


\section{Discussion}

In the work presented here we have demonstrated the potential of porcine bronchiole EVOCs for a model system for studying cellular trauma of tissue in the lower respiratory tract. Tissue explants of respiratory tissues are capable of maintaining many characteristics of in vivo physiology and cellular complexity in whole animals [10]. The shock tube system used in these studies enables one to tailor outputs to produce pressure loading that can be used to mimic features associated with scenarios that can result in injury patterns over a range of scales, from cells to whole animal models [7-9]. Our aim is to utilise these features to create meaningful, tissue-based models of mechanical trauma that can be used to study underlying tissue damage mechanisms associated with blast lung and ARDS.

Acute lung injury and ARDS can arise from a wide range of trauma-related events, often associated with blunt mechanisms and chest injury [11]. Immunopathophysiology of ARDS in respiratory tissues caused by severe tissue trauma tends to follow a classic pattern of destruction, inflammation, thrombosis and destruction [11]. In primary blast lung, the destruction phase includes parenchymal injury and haemorrhage, but a mechanistic understanding of the physical and biological factors which lead to tissue damage are not well understood [1]. Dissipation of the kinetic energy associated with the blast wave is thought to generate shear and stress waves that affect the structural integrity of respiratory tissues, eventually resulting in deformation and surface haemorrhage of lung tissue [1,12.13]. Rapid compression and expansion of air bubbles in tissues is also believed to contribute to the rupture of lower airway structures, alveoli, that can be a hallmark of acute injury $[1,14]$. In blast lung injury as well as other severe injuries affecting respiratory tissues, local and systemic inflammatory responses result $[1,11]$. Therapeutic approaches for treating respiratory tissue trauma are likely to focus on immune and/or inflammatory targets that can modulate the negative effects of inflammation as a means of reducing tissue damage and/or preventing progression to ARDS [1.11].

Future studies will focus on developing the model presented here as a screening platform for pre-clinical studies for ARDS-related therapies. A key concern of non-whole animal models of blast lung has been a lack of complexity [1]. EVOCs, unlike cell-based models, replicate many physiological features seen in respiratory tissues in whole animals. In particular, the three-dimensional structure and cell diversity found in EVOCs reproduces the respiratory mucosa [11]. Importantly, EVOCs have been used to study the effects of pharmacological agents and innate responses [11 and references therein]. Hence, given the relative ease of inducing controlled damage to EVOCs using the shock tube, there is considerable potential to identify molecular markers involved in damage responses and use that information to improve study designs at smaller (cell) and larger (whole animal) scales.

\section{Acknowledgements}

This work was conducted with support from the Royal British Legion Centre for Blast Injury Studies at Imperial College London. The invaluable technical support of the Cavendish Laboratory workshop and the Cambridge Veterinary Histopathology Laboratory is gratefully acknowledged by the authors.

\section{References}

1. T.E. Scott, E. Kirkman, M. Haque, I.E. Gibb, P. Mahoney, J.G. Hardman, Br. J. Anaesth. 118, 311 (2017)

2. Z. Sziklavari, T.F. Molnar, J. Thorac. Dis. 11(Suppl 2), S167 (2019)

3. G.J. Cooper, D. Townsend, J. R. Army Med. Corps 135, 58 (1989) 
4. W.G. Proud, J. R. Army Med. Corps 159(Suppl 1), i4 (2013)

5. J.E. Smith, J. Garner, J. R. Army Med. Corps. 165, 57 (2019)

6. B.J. Butler, A. Williams, A.W. Tucker, W.G. Proud, K.A. Brown, EPJ ST 227, 55 (2018)

7. T-T. Nguyen, A.P. Pearce, D. Carpanen, D. Sory, G. Grigoriadis, N. Newell, J. Clasper, A. Bull, W.G. Proud, S.D. Masouros, J. R. Army Med. Corps 165, 33 (2019)

8. T. Eftaxiopoulou, A. Barnett-Vanes, H. Arora, W. Macdonald, T-T.N. Nguyen, M. Itadani, A.E. Sharrock, D. Britzman, W.G. Proud, A.M.J. Bull, S.M. Rankin, Injury 47, 625 (2016)

9. B.J. Butler, C. Bo, A.W. Tucker, A.P. Jardine, W.G. Proud, A. Williams, K.A. Brown, JPCS 500, 182007 (2014)

10. S.F. Nunes, P.R. Murcia, L.S. Tiley, I.H. Brown, A.W. Tucker, D.J. Maskell, J.L. Wood, Influenza Other Respir. Viruses 4, 7 (2009).

11. L. Lupu, A. Palmer, M. Huber-Lang M, Front Immunol. 11, 584514 (2020).

12. G.J. Cooper, D. Townsend D. J. R. Army Med. Corps 135, 58 (1989).

13. G.J. Cooper, J. Trauma 40(3 Suppl), S105 (1996).

14. D.A. Rice, J. Appl. Physio. Respir. Environ. Exerc. Physiol. 54, 304 (1983). 